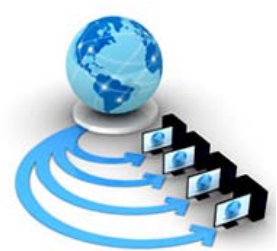

Volume 8, No. 7, July - August 2017

International Journal of Advanced Research in Computer Science

RESEARCH PAPER

\author{
Available Online at www.ijarcs.info
}

\title{
A COMPARISON OF ARTIFICIAL NEURAL NETWORK AND K-NEAREST NEIGHBOR CLASSIFIERS IN THE OFF-LINE SIGNATURE VERIFICATION
}

\author{
Jasmeet Kaur \\ Electronics and Engineering Department \\ Punjabi University, Patiala , India
}

\author{
Dr. Reecha Sharma \\ Electronics and Engineering Department \\ Punjabi University, Patiala ,India
}

\begin{abstract}
The ANN is a new classification technique within the field of applied mathematics learning theory that has been applied successfully in pattern recognition applications like signature, face and speaker recognition, whereas the k-NN may be a non parametric technique used for classification of handwriting recognition and signature verification. This paper reports on a comparison of the 2 classifiers in off-line signature verification. For this purpose, associate acceptable learning and testing protocol was created to watch the potential of the classifiers to soak up intrapersonal variability and highlight social similarity using random, easy and simulated forgeries by finding the accuracy.
\end{abstract}

Keywords: Classification; Artificial Neural network; k-Nearest Neighbours; Signature verification

\section{INTRODUCTION}

There are basically two issues underlying off-line signature verification. One is said to the quantity of samples to use for learning. In an exceeding real application, we are sometimes quite restricted within the range of samples we will use for training (4-6 samples). The other is that the ability of the system to discriminate among differing kinds of forgeries (random, easy and simulated).The random forgery is sometimes described by a genuine signature sample, that belongs to a different writer not essentially listed to the signature verification [1]. The simple forgery is described by a signature sample with a similar semantic of the genuine writers name with none conceive to imitate the genuine signature model. The simulated forgery is described by an affordable imitation of the genuine signature model. Any learning methodology that claims to resolve those issues depends on its ability to perform the training and classification tasks. Usually, in signature verification, 2 totally different pattern categories are required for the educational task, W1 and W2. W1 represents a genuine signature set. W2 represents a forged signature set. Within the latter case, the genuine signatures of various writers are used like random forgeries. For real applications, like order of payment authentication, easy and simulated forgeries don't seem to be employed in the training part [5].

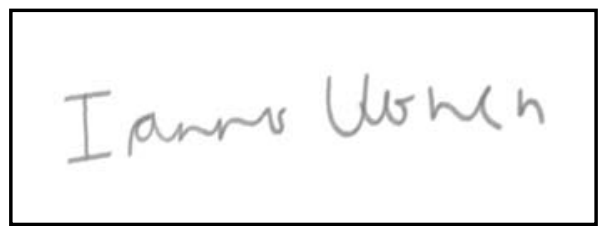

(a)

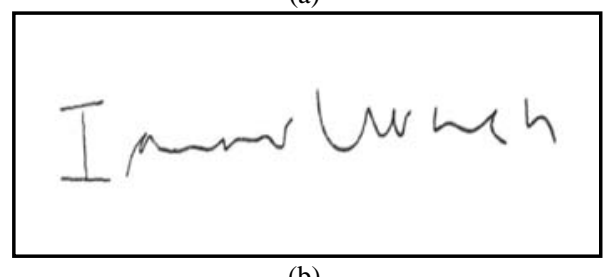

(b)

Figure 1: (a) Genuine and (b) forged signature
The most challenge within the learning task is to separate categories W1 and W2. In several cases, the brink between them isn't straightforward to search out. Within the verification task, the challenge is to discriminate between the genuine and every one variety of forgeries, having in mind for training solely a priori information of some random forgeries. This will increase the verification task quality particularly once simple and simulated forgeries are challenging the verification system, as Simulated forgeries, as an example, are just like real signatures in some ways that. For this reason, some samples work into the W1 category and a few nearly do so. This might even be the case with simple forgery samples. In this paper, a comparison between ANN and $\mathrm{k}-\mathrm{NN}$, in terms of the training and verification tasks described above, is conferred [1].

\section{SIGNATURE VERIFICATION AND k-NN}

The k-nearest neighbour classifier is viewed as distributing the $\mathrm{k}$ nearest neighbours a weight $1 / \mathrm{k}$ and every one others zero weight. This may be generalised to weight nearest neighbour classifiers. Given a group $\mathrm{X}$ of $\mathrm{n}$ points and $\mathrm{a}$ distance operate $\mathrm{D}$; k-nearest neighbour (k-NN) search permits you to search out the $\mathrm{k}$ nearest points in $\mathrm{X}$ to $\mathrm{a}$ question point or set of points. The $\mathrm{k}-\mathrm{NN}$ search technique and k-NN based mostly algorithms are frequently used as benchmark learning rules the relative simplicity of the k-NN search technique makes it simple to match the results from alternative classification techniques to $\mathrm{k}-\mathrm{NN}$ results. they need been employed in varied areas like bioinformatics, image process and data compression, document retrieval, pc vision, multimedia database, and selling information analysis. K-NN is employed in rummage around for alternative machine learning algorithms, like k-NN classification, native weighted regression, missing information imputation and interpolation, and density estimation [3]. 


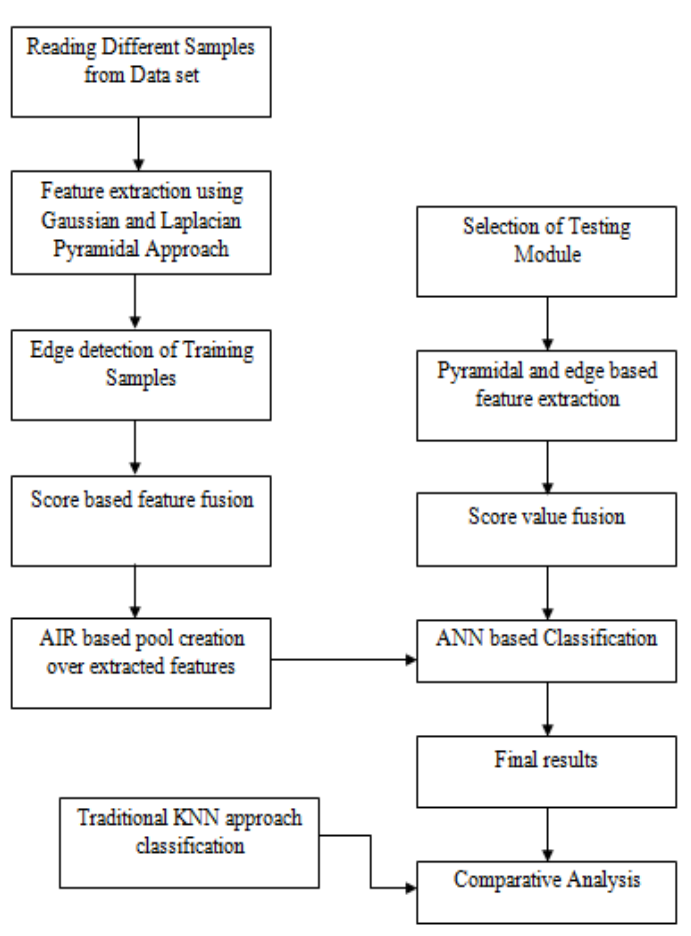

Figure 2: Block diagram of signature verification and comparison

We conjointly use k-NN search with several distance-based learning functions, like K-means cluster. In k-NN classification, the output could be a category membership. An object is classed by a majority vote of its neighbours, with the object being allotted to the category commonest among its $\mathrm{k}$ nearest neighbours ( $\mathrm{k}$ could be a positive whole number generally small). If $\mathrm{k}=1$, then the thing is solely allotted to the category of that single nearest neighbor [3].

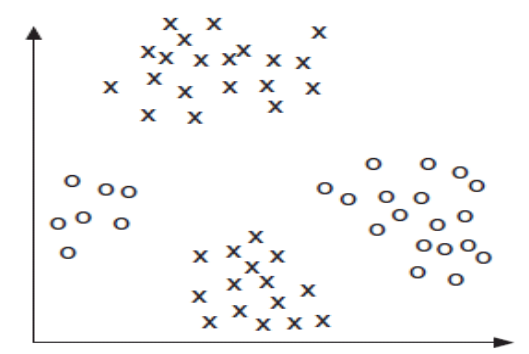

Figure 3: k - Nearest Neighbour clustering

\section{SIGNATURE VERIFICATION AND ANN}

A neural network model that is the branch of artificial intelligence is usually noted as artificial neural networks (ANNs). ANN teaches the system to execute task, rather than programming procedure system to try to definite tasks [6]. To perform such tasks, artificial intelligence System (AI) is generated. It's a practical model which may quickly and exactly realize the patterns buried in information that replicate helpful data. One case of those AI models is neural networks. AI systems ought to discover from knowledge on a continuing basis [7]. Within the areas of diagnosing relationships with dissimilar knowledge, the foremost accessible techniques are the artificial Intelligence techniques. a man-made neural network is formed of several artificial neurons that are correlative together in accordance with specific specification. The target of the neural network is to convert the inputs into vital outputs [4]. The teaching mode may be supervised or unsupervised. Neural Networks learn within the presence of noise. ANNs found their usage in several areas like, • Bankruptcy prediction • Speech recognition • Product review • Fault detection

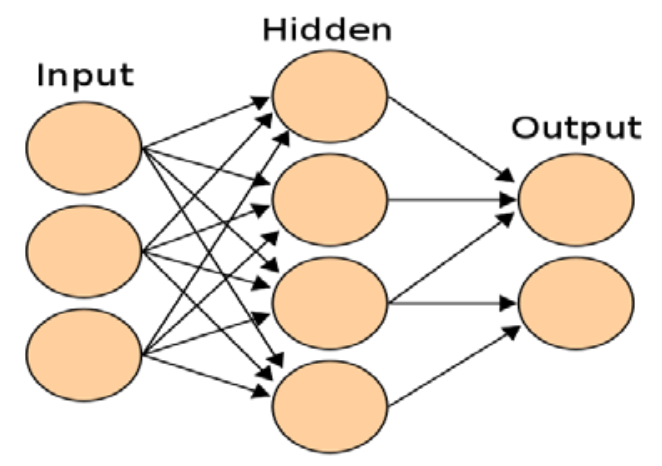

Figure 4: A neural network

\section{FEATURE EXTRACTION METHODS}

\section{Gaussian and Laplacian Pyramids}

The Gaussian pyramid is computed as follows. The first image is convolved with a Gaussian kernel. The resulting image could be a low pass filtered version of the first image. The cut-off frequency will be controlled employing the parameter $\sigma$. The Laplacian is then computed because the distinction between the first image and also the low pass filtered image. This method is sustained to obtain a group of band-pass filtered images (since each is the distinction between 2 levels of the Gaussian pyramid). Therefore the Laplacian pyramid could be a set of band pass filters [2].

Imagine these pictures stacked one on top of another; the result's a tapering pyramid system - therefore the name. The Laplacian pyramid will therefore be accustomed represent pictures as a series of band-pass filtered pictures, every sampled at in turn sparser densities. It's oftentimes utilized in image processing and pattern recognition tasks due to its easy computation. We were ready to extract the text from the background in a picture by using three levels of the Laplacian pyramid. We tend to use $\mathrm{k}$ mean cluster to phase the three pictures obtained at every level of the pyramid. The text, which contains a stronger response to the filters, forms one cluster, whereas the background areas with very little intensity variation type a separate cluster

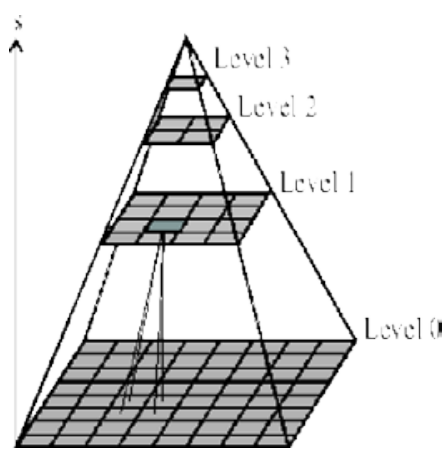

Figure 4: Tapering pyramid structure 


\section{Canny edge detector}

Canny edge detection is a technique to extract helpful structural data from totally different vision objects and dramatically scale back the quantity of knowledge to be processed. It's been wide applied in numerous pc vision systems. Canny has found that the necessities for the appliance of edge detection on numerous vision systems are comparatively similar. Thus, an edge detection answer to handle these necessities will be enforced in an exceedingly wide selection of things [8].

The general criteria for edge detection include:

-Detection of edge with low error rate, which implies that the detection ought to accurately catch as several edges shown within the image as attainable.

-The edge purpose detected from the operator ought to accurately localize on the middle of the edge.

-A given come on the image ought to solely be marked once, and wherever attainable, image noise mustn't produce false edges.

To satisfy these necessities canny used pure mathematics of variations a method that finds the function that optimizes a given functional. The optimum function in Canny's detector is represented by the total of 4 exponential terms; however it will be approximated by the primary by-product of a Gaussian [2].

Among the edge detection strategies developed thus far, canny edge detection algorithmic rule is one amongst the foremost strictly outlined strategies that gives smart and reliable detection. As a result of its optimality to fulfil with the 3 criteria for edge detection and therefore the simplicity of method for implementation, it became one amongst the foremost common algorithms for edge detection.

\section{EVALUATION PROTOCOL}

For ANN and k-NN database pd Scorpus was collected by Grupode Procesado Digital de Senales. The primary assortment contained signatures for a hundred writers with twenty four real and thirty trained forgeries. This results in 2400 real signatures and 3000 forgeries within the whole dataset. Real signatures were collected on constant day wherever signers crammed up a kind with twenty four boxes of various sizes [3]. Forgeries were collected on a variety of fifteen boxes by asking every forger to imitate three signatures of five every which way elite signers. Once signature forms were collected, every kind was scanned with a Canon device using 256 level gray scales. Thereafter, they were changed to black and white employing a global threshold that was computed using Otsu's technique. The ensuing binary image is then skilful a median filtering to eliminate normalise like minor discontinuities further as salt and pepper noises. The feature extracted victimization the Gaussian and Laplacian pyramid and edge detection we tend to extracted the results.

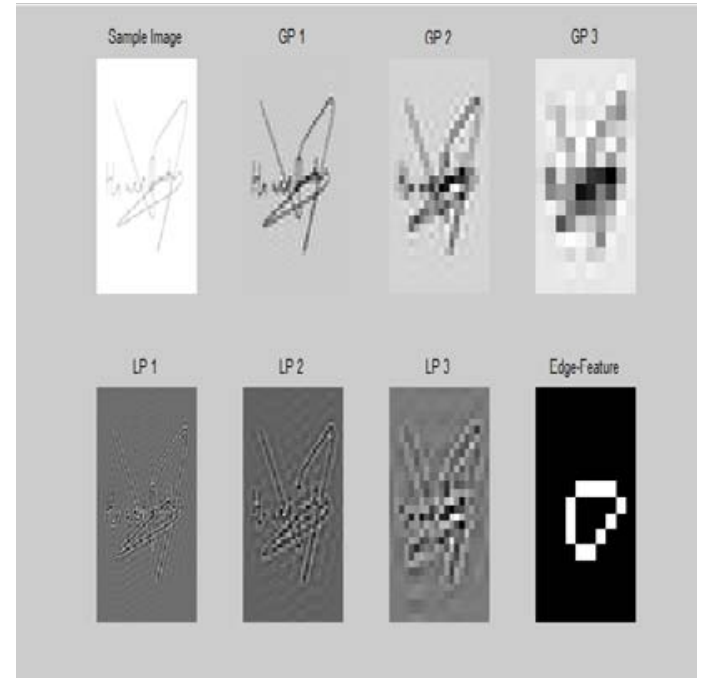

Figure5: Result of Feature extraction by Gaussian pyramid, Laplacian pyramid and edge detection

\section{EXPERIMENTAL RESULTS}

The accuracy and the percentage error is detected using both $\mathrm{k}-\mathrm{NN}$ and ANN classifier at the verification stage of signature and during the training stage AIR .The feature are extracted by using the Gaussian, Laplacian pyramid and canny edge detector and then score based result is formed and then they are passed to classifier to see the matched and non matched samples and the accuracy.

As we can see by using the ANN we get better accuracy up to $90 \%$ and by using the $\mathrm{kNN}$ the accuracy is $60 \%$. And the percentage error rate also of ANN is less than $10 \%$ and for k-NN its 38 \%approx.

Table 1: Result of percentage Error and Accuracy

\begin{tabular}{|c|c|c|}
\hline Classifier & $\begin{array}{c}\text { Percentage } \\
\text { error } \%\end{array}$ & Accuracy \% \\
\hline k- NN & 10 & 90 \\
\hline ANN & 38 & 60 \\
\hline
\end{tabular}

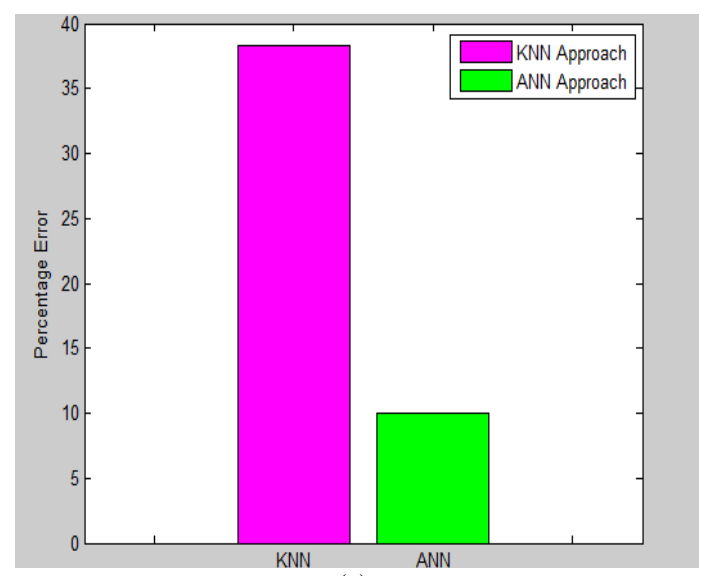

(a) 


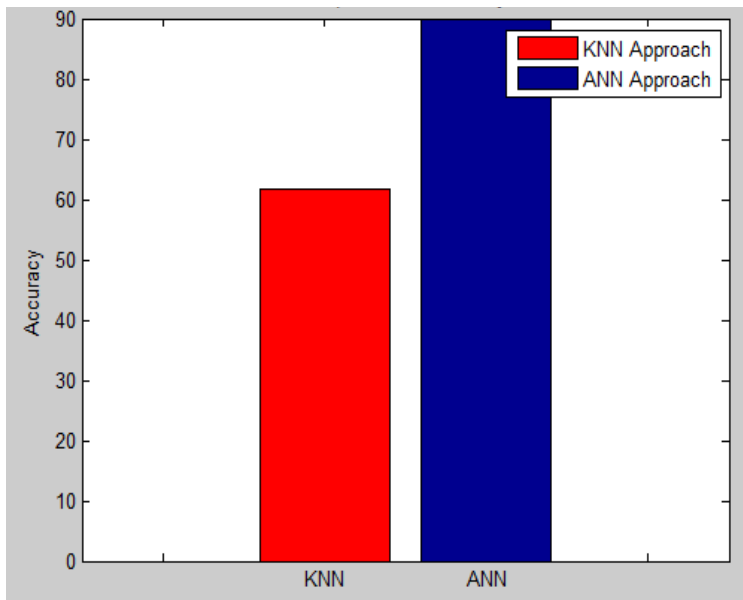

(b)

Figure 6: Result of (a) percentage error and (b) accuracy of k-NN and ANN

\section{CONCLUSION AND FUTURE WORK}

The main objective of this study was to match ANN and kNN classifiers beneath 2 specific conditions, the primary being the amount of samples used for training, and therefore the second being the utilization of various kinds of forgeries. Beneath each condition, the ANN showed higher results. However, in terms of random forgery acceptance and small range of samples accustomed training, the ANN showed promising results, demonstrating ANN ability to spot straightforward and simulated forgeries while not previous information.

In a future work are going to be enlarged the information, enforced new graph metric options and a replacement protocol with the most objective to reduces the amount of signature training samples and reduces the acceptation error rate in simulate forgery sort.

\section{REFERENCE}

[1] Justino, E.J., Bortolozzi, F. and Sabourin, R., 2005. A comparison of SVM and HMM classifiers in the off-line signature verification. Pattern recognition letters, 26(9), pp.1377-1385.

[2] Wang, W. and Chang, F., 2011. A multi-focus image fusion method based on Laplacian pyramid. JCP, 6(12), pp.25592566.

[3] Serdouk et al. "New off-line Handwritten Signature Verification method based on Artificial Immune Recognition System." Expert Systems with Applications 51 (2016): 186194.

[4] Choudhary et al. "Signature Recognition \& Verification System Using Back Propagation Neural Network." International Journal of IT, Engineering and Applied Sciences Research 2.1 (2013).

[5] Singh et al. "A Survey on Offline Signature Recognition and Verification Schemes." IOSR Journal of Electronics and Communication Engineering (IOSRJECE) ISSN: 2278-2834.

[6] Dewan et al. "Offline signature verification using neural network." International Journal Of Computational Engineering \& Management (IJCEM) 15.4 (2012): 50-54.

[7] Timmis et al. "A resource limited artificial immune system for data analysis." Knowledge-Based Systems 14.3 (2001): 121130.

[8] Canny, J., 1986. A computational approach to edge detection. IEEE Transactions on pattern analysis and machine intelligence, (6), pp.679-698. 